\title{
Constraining pulsar birth properties with supernova X-ray observations
}

\author{
Y. A. Gallant ${ }^{1}$, R. Bandiera ${ }^{2}$, N. Bucciantini ${ }^{2}$ and E. Amato ${ }^{2}$ \\ ${ }^{1}$ LUPM, U. de Montpellier, CNRS/IN2P3, \\ place E. Bataillon, 34095 Montpellier, France \\ email: gallant@in2p3.fr \\ ${ }^{2}$ INAF - Osservatorio Astrofisico di Arcetri, \\ Largo E. Fermi 5, I-50125 Firenze, Italy
}

\begin{abstract}
A large fraction of core-collapse supernovae are thought to result in the birth of a rotation-powered pulsar, which is later observable as a radio pulsar up to great ages. The birth properties of these pulsars, and in particular the distribution of their initial rotation periods, are however difficult to infer from studies of the radio pulsar population in our Galaxy. Yet the distributions of their birth properties is an important assumption for scenarios in which ultrahigh-energy cosmic rays (UHECRs) originate in very young, extragalactic pulsars with short birth periods and/or high magnetic fields.

Using a model of the very young pulsar wind nebula's dynamical and spectral evolution, with pulsar wind and accelerated particle parameters assumed similar to those inferred from modeling young pulsar wind nebulae (PWNe) in our Galaxy, we show that X-ray observations of supernovae, a few years to decades after the explosion, constitute a favored window to obtain meaningful constraints on the initial spin-down luminosity of the newly-formed pulsar. We examine the expected emerging PWN spectral component, taking into account the X-ray opacity of the expanding supernova ejecta, and find that it is typically best detectable in $<10 \mathrm{keV}$ X-rays some years after the explosion. We use this framework to assess available X-ray observations and flux upper limits on supernovae, building on the work of Perna et al. (2008). We note that a resulting limit on spin-down luminosity corresponds univocally to a limit on the maximum magnetospheric acceleration potential, irrespective of the specific combination of magnetic field and rotation period that achieves it. We use available X-ray observations of supernovae to place constraints on the birth spin-down luminosity and period distribution of classical pulsars. We also examine the case of magnetars, born with much higher magnetic fields, and show that their much shorter initial spin-down time implies that any plausible signature of young magnetar wind nebulae can only be observed in harder X-ray or gamma-rays.
\end{abstract}

Keywords. pulsars, supernovae, acceleration of particles, X-rays, neutron stars

\section{Introduction and motivation}

The inferred Galactic radio pulsar birth rate is a large, and possibly dominant, fraction of the Galactic core-collapse supernova rate, implying that many, and possibly most, such supernovae result in the formation of a rotation-powered pulsar. Radio pulsar population synthesis studies (e.g. Faucher-Giguère \& Kaspi 2006, and references therein) suggest that their surface magnetic fields $B_{*}$ follow a log-normal distribution, with $\left\langle\log B_{*} / \mathrm{G}\right\rangle \approx$ $12.5 \pm 0.5$; the distribution of birth periods $P_{0}$ is however poorly constrained by such studies, as pulsars "lose memory" of $P_{0}$ after their initial spin-down time $\tau_{0}$, which is of order kyr. Fast-spinning pulsars or magnetars have been proposed to power certain types of supernovae, but such scenarios currently lack an unambiguous observational signature 
(e.g., Chevalier 2011 and references therein). Here we propose to use a model of the "very young" wind nebulae powered by such pulsars to examine their potential observability.

\section{Dynamical and spectral model of very young PWNe}

Dynamical evolution. In the initial, so-called 'free expansion' phase of pulsar wind nebula (PWN) evolution, the wind of the newly-born pulsar blows a bubble of magnetized relativistic plasma inside the freely expanding ejecta of the supernova (e.g., van der Swaluw et al. 2001; Bucciantini et al. 2003). We assume that this wind carries most of the pulsar's spin-down power $\dot{E}$, and that the core of the ejecta, into which the PWN expands, has an approximately uniform density. In the first few years after the explosion, during which the pulsar's spin-down power remains approximately constant, the PWN's evolution can be described by an analytical solution in which its radius evolves as $R_{\text {pwn }} \propto t^{6 / 5}$ (Chevalier \& Fransson 1992 and references therein). We further assume that the magnetization fraction of the pulsar wind, $\eta$, remains constant and has a value similar to those inferred from models of 'young' PWNe with ages of order a few kyr; the median magnetization found in models of 9 young PWNe by Torres et al. (2014) was $\eta \approx 0.03$. Under the above assumptions, the PWN magnetic field in its early years is generally quite high:

$$
B_{\text {pwn }}\left(t_{\text {age }}\right) \approx 0.4 \mathrm{G} \times \sqrt{\frac{\eta}{0.03}}\left(\frac{E_{\text {ej }}}{10^{51} \mathrm{erg}}\right)^{-0.45}\left(\frac{\dot{E}}{10^{38} \mathrm{erg} / \mathrm{s}}\right)^{0.2}\left(\frac{t_{\text {age }}}{\mathrm{yr}}\right)^{-1.3},
$$

where $t_{\text {age }}$ is the supernova remnant's age and $E_{\text {ej }}$ the initial kinetic energy of the ejecta, which are assumed uniform with a fiducial mass of $5 M_{\odot}$. Strictly speaking, this expression assumes that radiative losses are dynamically unimportant, so that essentially all the pulsar's spin-down power can drive the expansion of the PWN. We will see below that this is not actually the case for very young PWNe, in which synchrotron losses are highly efficient. In the latter regime, the PWN magnetic field could be somewhat higher; estimates suggest that the correction factor remains of order unity, however, so we will use the magnetic field value from (2.1) in what follows.

Accelerated particle spectrum. Multi-wavelength observations of PWNe suggest that the relativistic electrons and positrons injected by the pulsar wind are accelerated to a broken power-law spectrum. In sufficiently young and magnetized PWNe, the maximum accelerated particle energy is reached when the synchrotron cooling time $t_{\text {cool }}$ becomes shorter than the acceleration time $t_{\text {acc }}$, and observations of the high-energy cutoff of the Crab Nebula's synchrotron spectrum suggest that $t_{\text {acc }}$ can be of order the particle's gyro-time (de Jager \& Djannati-Ataï 2009 and references therein). In very young PWNe, the resulting acceleration time scale is much shorter than the dynamical time $t_{\text {age }}$ :

$$
t_{\text {acc }}\left(\gamma_{\max }\right) \approx \frac{m_{e} \gamma_{\max } c}{e B_{\text {pwn }}\left(t_{\text {age }}\right)} \approx 25 \mathrm{~s} \times\left(\frac{\eta}{0.03}\right)^{-0.75}\left(\frac{t_{\text {age }}}{\mathrm{yr}}\right)^{1.95},
$$

with weaker dependencies on $\dot{E}$, the ejecta mass $M_{\mathrm{ej}}$ and $E_{\mathrm{ej}}$. The acceleration process should thus operate efficiently, radiating synchrotron photons up to high-energy $\gamma$-rays.

Typical accelerated $e^{ \pm}$spectral indices, defined by $\mathrm{d} N_{e} / \mathrm{d} \gamma \propto \gamma^{-p}$ where $\gamma$ is the particle Lorentz factor, are $p_{1} \approx 1.5$ and $p_{2} \approx 2.5$ for the low- and high-energy part of the (uncooled) spectrum, respectively, as shown by the median values of the PWN models of Torres et al. (2014). The break energy between the two power-law segments can differ significantly between PWNe, but a representative value is $\gamma_{\mathrm{br}} \simeq 3 \times 10^{5}$, the median value from the models of Torres et al. (2014). With the magnetic field (2.1), the 
corresponding (injection) break frequency in the synchrotron spectrum is given by:

$$
h \nu_{\mathrm{br}} \approx 0.2 \mathrm{keV} \times\left(\frac{\gamma_{\mathrm{br}}}{3 \times 10^{5}}\right)^{2} \sqrt{\frac{\eta}{0.03}}\left(\frac{E_{\mathrm{ej}}}{10^{51 \mathrm{erg}}}\right)^{-0.45}\left(\frac{t}{\mathrm{yr}}\right)^{-1.3} .
$$

Emitted synchrotron spectrum. The high magnetic field value implies rapid synchrotron cooling of the accelerated $e^{ \pm}$; indeed, the spectrum injected in the nebula remains essentially unaffected by radiative losses only below the synchrotron cooling break frequency, defined by $t_{\text {cool }}=t_{\text {age }}$ and given by

$$
\nu_{\text {cool }} \approx 1.2 \times 10^{10} \mathrm{~Hz} \times\left(\frac{\eta}{0.03}\right)^{-1.5}\left(\frac{E_{\mathrm{ej}}}{10^{51} \mathrm{erg}}\right)^{1.35}\left(\frac{t}{\mathrm{yr}}\right)^{1.9}
$$

ignoring the weaker dependencies on $\dot{E}$ and $M_{\mathrm{ej}}$. As $\nu_{\text {cool }} \ll \nu_{\mathrm{br}}$, the bulk of the synchrotron spectrum is 'cooled', with its peak in $\nu F_{\nu}$ at $\nu_{\mathrm{br}}$, and essentially all the accelerated particle energy is radiated away quasi-instantaneously (i.e. on a time scale short relative to the dynamical time $t_{\text {age }}$, although long relative to the acceleration time $\left.t_{\text {acc }}\right)$. For the particle spectral indices given above, the synchrotron spectral indices (defined by $F_{\nu} \propto \nu^{-s}$ ) are $s_{1} \approx 0.75$ and $s_{2} \approx 1.25$ below and above $\nu_{\text {br }}$, respectively, and the flux value at $\nu_{\mathrm{br}}$ is given by $\nu_{\mathrm{br}} F_{\nu_{\mathrm{br}}} \approx \frac{1}{8} \dot{E}$ (assuming $\eta \ll 1$ and $\left.\nu_{\text {cool }} \ll \nu_{\text {br }} \ll \nu_{\text {max }}\right)$. With all the above assumptions and fiducial parameter values, the fraction of the total pulsar spin-down power which is radiated in X-rays by the PWN is given by $L_{2-10 \mathrm{keV}} \approx 0.09 \dot{E}(t / \mathrm{yr})^{-0.325}$.

$X$-ray absorption by the ejecta. The X-rays emitted by the PWN are initially absorbed by the surrounding ejecta, and only become observable once these expanding ejecta become optically thin. The column density of the ejecta is equivalent to

$$
N_{\mathrm{H}}=4 \times 10^{24} \mathrm{~cm}^{-2} \times\left(\frac{M_{\mathrm{ej}}}{5 M_{\odot}}\right)^{2}\left(\frac{E_{\mathrm{ej}}}{10^{51} \mathrm{erg}}\right)^{-1}\left(\frac{t}{\mathrm{yr}}\right)^{-2},
$$

and we estimate the corresponding spectral absorption using the phabs model of interstellar X-ray absorption (Bałucińska-Church \& McCammon 1992). Representative emerging PWN X-ray spectra at different ages, emitted as described earlier and absorbed as outlined above, are shown in Figure 1. It can be seen that PWN X-rays in the energy range below $10 \mathrm{keV}$ typically only become observable a few years after the supernova, although harder X-rays can emerge earlier.

It should be noted that the X-ray absorption model we use is only an approximation, as it assumes the absorbing medium is neutral, while the ejecta are at least partially ionized at the ages of interest. The phabs model should nonetheless remain roughly applicable, as its dominant process is photoelectric absorption of K-shell electrons, which remains effective unless the ejecta ions are fully stripped. More importantly, in normalizing to the absorbing column (2.5) we implicitly assume a hydrogen-dominated composition and Galactic interstellar abundances; ejecta significantly enriched in heavy elements would yield correspondingly higher absorption.

\section{Constraints on pulsar $\dot{E}_{0}$ and $P_{0}$ from observations}

X-ray observations of supernovae. Perna et al. (2008) used archival X-ray observations of extragalactic core-collapse supernovae (SNe), generally performed years or decades after the explosion, to constrain the initial spin-down power $\dot{E}_{0}$ of any embedded pulsar. Here we improve the interpretation of their upper limits by using the physically-derived PWN spectral model described above instead of their empirical X-ray luminosity relation. 


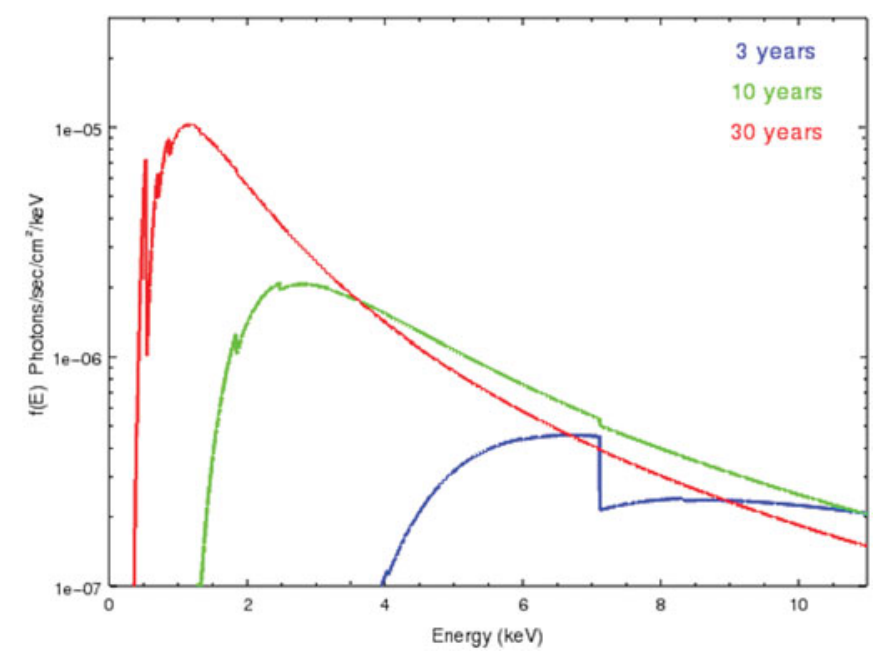

Figure 1. Estimated emerging PWN X-ray spectra, for the model and parameter values described in the text, at $t_{\text {age }}=3,10$ and 30 years after the supernova explosion.

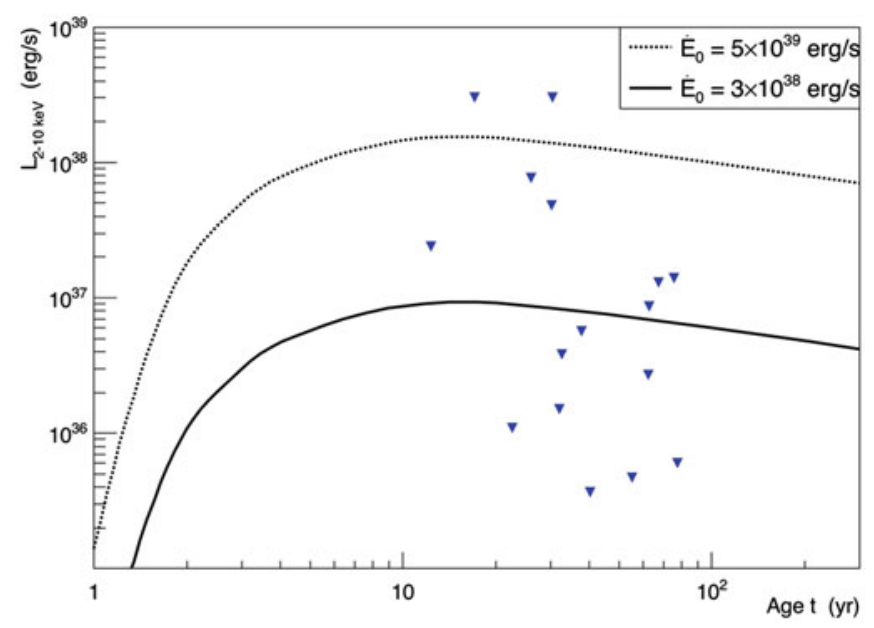

Figure 2. Estimated emerging PWN X-ray fluxes for the model and parameter values detailed in Sect. 2 and two representative values of $\dot{E}_{0}$, along with upper limits from Perna et al. (2008).

Figure 2 shows emerging PWN X-ray fluxes as a function of age, as predicted by the model described in Section 2, along with Perna et al.'s (2008) late-time (>10yr) flux upper limits on SNe of type IIP. (As type IIP SNe make up the dominant fraction of corecollapse $\mathrm{SNe}$, it is reasonable to suppose that at least a significant fraction of them give birth to pulsars.) As can be seen in Fig. 2, roughly $50 \%$ of the upper limits conflict with the predictions of the model for a spin-down luminosity of $\dot{E}_{0}=3 \times 10^{38} \mathrm{erg} / \mathrm{s}$ (similar to the Crab's), and $\sim 90 \%$ conflict with $\dot{E}_{0}=5 \times 10^{39} \mathrm{erg} / \mathrm{s}$ (an order of magnitude higher).

Under the assumption that these SNe gave birth to pulsars, the above can be interpreted as upper limits on the fraction of pulsars born with high spin-down luminosities. A pulsar's spin-down luminosity can be related to its rotation period and surface magnetic field through the standard magnetic dipole radiation formula:

$$
\dot{E} \equiv 3.3 \times 10^{40} \mathrm{erg} / \mathrm{s}\left(\frac{B_{*}}{3 \times 10^{12} \mathrm{G}}\right)^{2}\left(\frac{P}{10 \mathrm{~ms}}\right)^{-4}
$$


For a typical radio pulsar magnetic field value of $3 \times 10^{12} \mathrm{G}$, the above limits would thus correspond to $\sim 50 \%$ of such pulsars born with initial periods longer than $\sim 30 \mathrm{~ms}$, and $\sim 90 \%$ with initial periods longer than $\sim 15$ ms.

Other constraints. An alternative approach exploits the fact that the wind nebulae of young and powerful extragalactic pulsars would contribute to the population of ultraluminous X-ray sources (ULXs) to obtain constraints on their birth periods (Medvedev \& Poutanen 2013, and references therein). These authors find, using the observed X-ray luminosity distribution of ULXs, that the average birth period $P_{0}$ of radio pulsars must be longer than $\sim 10-40 \mathrm{~ms}$, in agreement with the constraints derived above.

\section{Applicability to magnetars}

Magnetars are neutron stars whose surface magnetic fields, as inferred from their spindown through the magnetic dipole formula (3.1), have typical values $B_{*} \sim 10^{14}-10^{15} \mathrm{G}$, much higher than those of the rotation-powered, radio pulsars which we considered thus far. Magnetars further distinguish themselves by generally being radio-quiet, and by the fact that their observed emission is thought to be powered in large part by magnetic field decay rather than rotation.

Magnetars born with a very short rotation periods, of order a few milliseconds, have been proposed as the 'engine' of some classes of superluminous supernovae and gammaray bursts (e.g. Metzger et al. 2011, and references therein). It has also been proposed that ultra- high-energy cosmic rays (UHECRs) could originate in such fast-spinning magnetars (e.g., Blasi et al. 2000; Arons 2003; Fang et al. 2012; Lemoine et al. 2015). That magnetars should be born with short rotation periods is in fact expected in the dynamo theory of magnetar formation (Duncan \& Thompson 1992). The question thus arises whether newly-formed, powerful magnetars formed in supernovae might yield a highenergy observational signature similar to that discussed above for very young pulsar wind nebulae.

Early spin-down of magnetars. Under the magnetic dipole spin-down assumption, a newly-formed pulsar or magnetar retains memory of its initial rotation period for a time of order its initial characteristic spin-down time,

$$
\tau_{0} \approx 180 \mathrm{yr}\left(\frac{B_{*}}{3 \times 10^{12} \mathrm{G}}\right)^{-2}\left(\frac{P_{0}}{10 \mathrm{~ms}}\right)^{2} .
$$

For the classical rotation-powered radio pulsars considered thus far, $\tau_{0}$ is thus of order centuries or at least decades, implying that the initial spin-down luminosity remains approximately constant until the supernova ejecta become transparent to X-rays. For the much higher magnetic fields typical of magnetars, by contrast, $\tau_{0}$ is of order days, or even less for very short birth periods $P_{0}$.

It follows that newly-born fast magnetars release their considerable rotational energy very early, well before the ejecta become optically thin to X-rays. For ages $t \gg \tau_{0}$, a neutron star's spin-down evolution is solely determined by its magnetic field $B_{*}$, higher $B_{*}$ corresponding to lower $\dot{E}$ at a given age. Thus by the time the ejecta become transparent to X-rays, any observational signature of a magnetar's spin-down could no longer yield an upper limit on its initial rotation frequency.

It is moreover unclear whether one should expect magnetars to be surrounded by wind nebulae analogous to those described in Section 2; no 'magnetar wind nebulae' have been detected around the known, slower-spinning magnetars, with one plausible exception (Younes et al. 2016, and references therein). Nonetheless assuming that fastspinning magnetars as well as classical pulsars give rise to similar wind nebulae, Murase 
et al. (2015) made predictions for their emerging spectra at supernova ages of order one year. They found that synchrotron emission from such nebulae should be detectable in the hard X-ray domain (at energies of several tens of $\mathrm{keV}$ ), in which the ejecta become optically thin earlier, given the sensitivity of an instrument such as NuSTAR.

\section{Summary}

Classical radio pulsars $\left(B_{*} \sim 10^{12}-10^{13} \mathrm{G}\right)$. Extrapolating models of young pulsar wind nebulae and their parameter values back to the first several years after the supernova explosion, we find that such 'very young' PWNe efficiently radiate the newly- born pulsar's spin-down power $\dot{E}$. The emitted synchrotron radiation typically peaks (in $\nu F_{\nu}$ ) at photon energies just below X-rays. At ages less than several years, this radiation is essentially fully absorbed in the supernova ejecta, and can thereby contribute to powering the supernova light curve.

At ages of several years to decades, the ejecta gradually become transparent to Xrays, in which the very young PWN can thus be directly observed. X-ray upper limits on supernovae observed more than a decade after the explosion then constrain the birth power $\dot{E}_{0}$ of pulsars. For typical magnetic field values, available limits are consistent with a median birth period $P_{0} \gtrsim 30 \mathrm{~ms}$, and suggest that no more than $\sim 10 \%$ of pulsars could be born with periods shorter than $\sim 15 \mathrm{~ms}$.

Magnetars $\left(B_{*} \sim 10^{14}-10^{15} \mathrm{G}\right)$. Their much stronger magnetic fields imply that newlyformed magnetars spin down much earlier after birth than classical radio pulsars, with characteristic spin-down times $\tau_{0}$ of order days rather than centuries. They will thus have lost most of their initial rotation energy, and any memory of their birth period, well before the ejecta can become transparent to X-rays at energies $\lesssim 10 \mathrm{keV}$. Very young magnetar wind nebulae, if they exist, may however be detectable in hard X-rays.

\section{References}

Arons, J. 2003, ApJ, 589, 871

Bałucińska-Church, M. \& McCammon, D. 1992, ApJ, 400, 699

Blasi, P., Epstein, R. I., \& Olinto, A. V. 2000, ApJ (Letters), 533, L123

Bucciantini, N., Blondin, J. M., Del Zanna, L., \& Amato, E. 2003, A\&6A, 405, 617

Chevalier, R. A. 2011, AIP Conf. Proc., 1379, 5 (arXiv:1011.3731)

Chevalier, R. A. \& Fransson, C. 1992, ApJ, 395, 540

de Jager, O. C. \& Djannati-Ataï, A. 2009, in: W. Becker (ed.), Neutron Stars and Pulsars (Berlin and Heidelberg: Springer-Verlag), p. 451 (arXiv:0803.0116)

Duncan, R. C. \& Thompson, C. 1992, ApJ (Letters), 392, L9

Fang, K., Kotera, K., \& Olinto, A. V. 2012, ApJ, 750, 118

Faucher-Giguère, C.-A. \& Kaspi, V. M. 2006, ApJ, 643, 332

Lemoine, M., Kotera, K., \& Pétri, J. 2015, JCAP, 07, 016 (arXiv:1409.0159)

Medvedev, A. S. \& Poutanen, J. 2013, MNRAS, 431, 2690

Metzger, B. D., Giannios, D., Thompson, T. A., Bucciantini, N., \& Quataert, E. 2011, MNRAS, 413, 2031

Murase, K., Kashiyama, K., Kiuchi, K., \& Bartos, I. 2015, ApJ, 805, 82

Perna, R., Soria, R., Pooley, D., \& Stella, L. 2008, MNRAS, 384, 1638

Torres, D. F., Cillis, A., Martín, J., \& de Oña Wilhelmi, E. 2014, J. High Energy Astrophys., 1, 31 (arXiv: 1402.5485)

van der Swaluw, E., Achterberg, A., Gallant, Y. A., \& Tóth. G. 2001, A\& A, 380, 309

Younes, G., Kouveliotou, C., Kargaltsev, O., Gill, R., Granot, J., et al. 2016, ApJ, 824, 138 